\title{
Decision support for optimal adaptation of product and supply chain systems based on real options theory
}

\author{
Chong Hyun Park, Lalit Patil, Kazuhiro Saitou, Senior Member, IEEE, and Edwin Romeijn
}

\begin{abstract}
In order to remain profitable in the highly competitive global market, a manufacturing enterprise is expected to proactively adapt itself in anticipation of unplanned, but foreseeable high impact events. This paper presents a decision model for optimal adaptation of product and supply chain systems subject to sudden, severe changes in the operating environment. Extending our previous work [1], decision-tree based framework is developed for optimally choosing design-supplier alternatives in multi-product, multi-echelon product and supply chain systems based on the real options theory. A case study on a two-component, two-echelon supply chain is presented which highlights unique characteristics of product quality as compared to cost and time.
\end{abstract}

\section{INTRODUCTION}

$\mathrm{M}$ anufacturing enterprises are operating in a globally distributed manner in the highly competitive global markets. However, this exposes the enterprise to new uncertainties with high impacts on its product design and supply chains. These include events, such as natural disasters, new technologies, changes in environmental regulation, and other uncertainties [2].

One advantage of a virtual enterprise is that it could adapt to such uncertainties by using the flexibility provided through multiple product designs and large (global) set of suppliers. This is like modularity, which enables adaptability by interchangeable modules with different functionalities, in product design. However, currently, adaptation in a virtual enterprise is mostly reactive in response to the variations that have already occurred. In order to remain profitable, it is desirable to adapt proactively in anticipation of unplanned, but foreseeable high impact events.

The real options theory provides a framework to evaluate flexibility and enable effective decision-making. A real option is the ability, without an obligation, to implement an alternative [3]. Calculating the option value of an alternative

Manuscript received March 05, 2009. Patil was supported in part by the University of Michigan's PLM Alliance and National Science Foundation award CMMI-0833839.

Chong-Hyun Park (email: chypark@umich.edu) and Edwin Romeijn (email: romeijn@umich.edu) are with the Industrial and Operations Engineering Department, and Lalit Patil (phone: +1-734-763-5871; fax: +1734-647-3170; email: lpatil@umich.edu) and Kazuhiro Saitou (email: kazu@umich.edu) are with the Mechanical Engineering Department at the University of Michigan, Ann Arbor, MI 48109, USA. independent of other alternatives could help us in determining which alternative is better, and possibly, also the right time to invest in it. However, it does not help us in making the typical decisions, e.g., deferring, switching among multiple alternatives, or abandoning at various time states, in the supply chain. In addition, decision-making in the real world is path dependent, i.e., the decision on an alternative at some future date may depend on the path taken by another alternative until then. Thus, we need to consider the alternatives as part of one "enterprise-level" system, and then apply real options "in" this system. This approach has also been advocated by [4] to design for flexibility. Thus, in this research, our goal is not to value the option, but to use the fundamental idea, i.e., the option value maximizes the future returns, to determine decisions along the time line.

In particular, we focus on the simultaneous consideration of the product design characteristics (quality) and the supply chain characteristics (cost and lead time) in adapting the product design or the supply chain, or both, i.e., in treating product design and its supply chain as a system.

The remainder of the paper is organized as follows: Section II discusses relevant work. Section III presents the overall model of the product design and supply chain system. Section IV briefly discusses the real options approach to evaluate the flexibility and derive decisions for optimal adaptation. Section V details the application to a two-component two-echelon product design and supply chain system. Section VI discusses the unique characteristics and challenges posed by the inclusion of product quality as a performance driver. Section VII presents computational results of application to two scenarios. Finally, the paper concludes with a discussion for future work in Section VIII.

\section{RELATED WORK}

Most studies on how an enterprise adapts disruptions have focused on events that have already occurred.

In [5], a LP formulation with a deterministic model is used to capture the resilience in the manufacturing enterprise when the company meets disruptive events.

Models to design supply chains that perform well when the variations are beyond the limits of robustness are presented in [6]. It states that supply chain optimization models have traditionally assumed certainty of information, and argues the need for models that incorporate uncertainty into strategic decisions about supply chain design.

In [7], the authors focus on designing a six-sigma supply chain that aims for delivery times within the $6 \sigma$ limits, i.e., on robustness of achieving the delivery time. They do not 
consider the costs or other criteria. In [8], the authors discussed choosing suppliers to minimize the expected shortfall under disruption. The overall idea is to match demand and supply using only costs as the criteria.

The concept of real options is studied to model flexibility in an uncertain environment and has been used for decision making in several areas. Identifying possible real options in the engineering system such as project management is presented in $[10,11]$. A systematic approach to solve the path-dependency and interdependency features is presented that enables a plan development for the executions of projects. Dual-sourcing strategies with real option analysis are presented in [12] that mitigate the impact of disruptions in supply chains. The model also demonstrates that the value of the option of delaying a decision works best by using a dynamic programming approach. A supply chain model that enables the selection of suppliers, plant location, and market regions considering an implementation time lag for the supply chain operations for a manufacturing firm is presented in [13]. The real option approach is implemented to estimate the value of the flexibility and to determine the optimal strategy when the currency exchange rate changes.

This paper builds upon our initial work reported in [1], that presented the concept of adaptation, the need to use real options framework, and the application to a simple singlecomponent single-echelon product design and supply chain system. In [1], quality was included as a driver of performance, but it was treated in the same way as cost and time. In this paper, we attempt to understand the adaptation of and develop extensions to a two-component, two-echelon supply chain. In particular, we emphasize on the primary challenge that unlike cost and lead time, product quality is usually not a simple linear combination the constituent component qualities.

\section{OVERALL MODEL OF PRODUCT DESIGN AND SUPPLY CHAIN SYSTEM}

We propose a product design and supply chain system to be modeled as a triple $(D, S, E)$, where $D$ is the design of the product consisting of $n$ components, $S$ is a set of suppliers for each component, and $E=\left\{\left(S_{i}, S_{j}\right) \mid S_{i}, S_{j} \in S\right\}$ represents the connections among suppliers in $S$, i.e., supply chain. We shall refer to $\left(D_{i}, S_{i}\right) \in D \times S, 1 \leq i \leq n$ as componentsupplier subsystems. Here, a component simply refers to an output from a supplier, which can be incomplete requiring further processing by other supplier(s).

We consider the scenario in which the product design and supply chain system needs to adapt to a foreseeable, but high impact change in the environmental regulation, such as change in the amount of Hexavalent Chromium allowed in the product.

\section{A. Performance of the product and supply chain system}

Let $R_{i}(t)$ be the ability of component-supplier subsystem $\left(D_{i}\right.$, $\left.S_{i}\right)$ to meet the requirement (e.g., substance regulation) during $[t, t+\Delta t]$. Then, $Q_{i}, C_{i}$, and $T_{i}$, the abilities of $\left(D_{i}, S_{i}\right)$ to meet the quality, cost, and lead time requirements during $[t$, $t+\Delta t$, respectively, are functions of $R(t)$ and $t$. The overall performance of $(D, S, E)$ is some aggregation of $Q_{i}(t, R(t))$,
$C_{i}(t, R(t))$, and $T_{i}(t, R(t))$ for every component-supplier subsystem $\left(D_{i}, S_{i}\right)$ :

$P(t, R(t))=f(Q(t, R(t)), C(T, R(t)), T(t, R(t)))$

where,

$Q(t, R(t))=g\left(Q_{1}\left(t, R(t), Q_{2}(t, R(t)), \ldots, Q_{n}(t, R(t))\right)\right.$

$\left.C(t, R(t))=C_{1}(t, R(t))+\ldots+C_{n}(t, R(t))\right)+$ const

$T(t, R(t))=h\left(T_{1}(t, R(t)), \ldots T_{n}(t, R(t)), E\right)$

Function $f$ in Equation (1) is some aggregation of the overall quality, cost and time obtained from the componentsupplier subsystem. In this paper, we assume that this is obtained as an average as follows:

$$
P(t, R(t))=\text { average }(Q(t, R(t)), C(T, R(t)), T(t, R(t)))
$$

Whereas the total cost (Equation (3)) is a sum of the costs for the component-supplier subsystems, the quality and time are not. Functions $g$ and $h$ in Equations (2) and (4) depend on product design and topology of supply chain, respectively. The total lead time of an entire supply chain (and therefore its ability to meet the requirement) can typically be obtained by summing the lead times of supplier along each serial chain and taking the maximum of all parallel chains. For product quality, on the other hand, the situation is not as simple, as discussed in Section VI..

\section{B. Jump-diffusion model of the underlying processes}

The discrete disruptive events, which can occur within any product-supply chain system, are modeled by using jump processes [9]. We assume that the shock is transmitted to the ability of each of quality $(Q)$, cost $(C)$, and lead-time $(T)$ in the component-supplier subsystems. This is modeled as a geometric mean-reverting jump-diffusion process with Poisson shock: arrival rate, $\lambda$, and jump size, $\emptyset(0 \leq \emptyset \leq 1)$ :

$d X^{w}=\eta^{w}\left(\mu^{w}-X^{w}\right) X^{w} d t+\sigma^{w} X^{w} d z^{w}-X^{w} d q$

where, $w \in\{1,2,3\} ; X^{1}=Q, X^{2}=C$, and $X^{3}=T, \eta^{w}$ is the rate of return to the mean ability $\mu^{w}$ and $\sigma^{w}$ represents the volatility. These parameters can be obtained from archival data. In addition, $d z^{w}$ is a standard Brownian motion with covariance $\left[d z^{v}, d z^{w}\right]=\rho^{v w} d t$, and independent of $d q$. Thus, the Equation says that $X^{w}$ will fluctuate as a mean reverting process with mean, $\mu^{w}$ and variance, $\sigma^{w}$, but over each time interval, $d t$, there is a small probability, $\lambda d t$ that it will drop to $(1-\varnothing)$ times its original value, after which it continues fluctuating until another event occurs.

\section{REAL OPTIONS ANALYSIS}

The manufacturer's goal is to maximize the system's ability to meet its expected ability. The basic idea is to compare the ability to meet the performance target of the alternative product and supply chain systems, to the manufacturer's expected ability and then choose the alternative with the highest payoff. This must include a flexible decision-making process along time, i.e., the decision to implement or continue holding the option is made at various time instants along the time horizon.

The reader is referred to [3] for details on the application of different stochastic programming approaches to solve real options problems. In our research, we work on the event tree 
that captures the variation of the manufacturer's expectation of the system's ability. At each node in the event tree, optimal decisions among abandonment, continuation, or switch to an alternative can be obtained by using a dynamic programming approach. The aim is to choose the decision variable $v_{t j} \in\{-1,0,1, \ldots, m\}$ at each node $(t, j)$ such that the value of the expected payoffs at time $t=0$ is maximized. Possible decisions, each with an opportunity cost, are as follows:

- Hold decision $\left(v_{t j}=0\right)$ and wait until next time step.

- Abandon project $\left(v_{t j}=-1\right)$ with salvage value $A$.

- Do not adapt $\left(v_{t j}=v_{t-1, j}\right)$ with no cost.

- Adapt to the $\boldsymbol{i}^{\text {th }}$ alternative product-supply chain $\operatorname{system}\left(v_{t j}=i\right)$ with switching cost, $K_{i}$.

An optimal value of the decision variable $v_{t j}$ can be obtained by solving the following stochastic recurrence equation:

$$
F_{t j}=\max _{v_{t j}}\left\{\pi\left(t, j, v_{t j}\right)+\frac{E_{t}\left(F_{t+1, j}\right)}{1+\gamma}\right\}
$$

where $F_{t j}$ is the total value of all the future payoffs at node ( $t$, $j$ ) if the firm follows the optimal decision after $t, \gamma$ is the discount rate, and $\pi\left(t, j, v_{t j}\right)$ is the difference between the alternative system's ability to meet the performance target and the manufacturer's expected ability. Details on the approach and application to this area can be obtained in [1].

Whereas the approach for real options analysis is the same for different supply chain topologies, the primary differences are: generation of the event trees and aggregation of cost, time, and quality as performance drivers.

The next section discusses the development of appropriate decision models in multi-echelon product design and supply chain system with a two-component product.

\section{APPLICATION TO TWO-COMPONENT TWO-ECHELON PRODUCT DESIGN AND SUPPLY CHAIN SYSTEM}

This section investigates the case in Figure 1, where two suppliers provide (complete) components to another supplier where they get "assembled" to a product. Each componentsupplier subsystem $\left(D_{i}, S_{i}\right), \quad i \in\{1,2\}$ has some alternative subsystem. In this configuration, two suppliers, $S_{1}$ and $S_{2}$, produce (incomplete) components, $D_{1}$ and $D_{2}$. The supplier (assembler), $S_{3}$, receives the components and forms the end product.

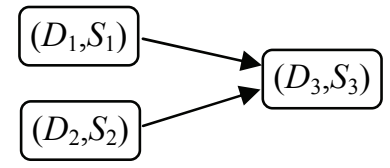

Figure 1 Two-echelon supply chain with two-component product.

A. Generating events trees for component-supplier
subsystem

We construct a discretized event tree using a trinomial expansion to capture the underlying process of $X^{w}$ for each component-suppler subsystem. This process is explained in detail in [1] and is summarized here. Figure 2 shows the possible events during the time interval $[t, t+\Delta t]$ :
- Scenario A (top): $X^{w}$ goes up by factor $u$ with probability $q_{t}^{+}$due to random fluctuation.

- Scenario B (middle): $X^{w}$ goes down by $d$; probability $q_{t}^{-}=$ $\left(1-q_{t}^{+}\right)$.

- Scenario C (bottom): $X^{w}$ goes down significantly by factor $1-\varnothing$ with probability $\lambda \Delta t$ due to a jump.

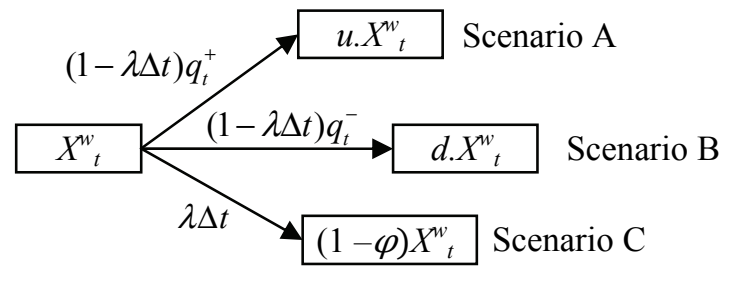

Figure 2 Three possible events in the interval $[t+\Delta t]$

Assuming that there is a 50-50 chance of scenarios A and $\mathrm{B}$ at $t$ in the absence of a jump, i.e., $q_{t}^{-}=q_{t}^{+}=0.5$ and $X^{w}$ follows a simple jump-diffusion process as in Equation (6). Then $u$ and $d$ are given as:

$$
\begin{aligned}
& u=\left(E\left[X_{t+\Delta t}^{w}\right]+\sqrt{\operatorname{Var}\left[X^{w}\right]}\right) / X_{t}^{w} \\
& d=\left(E\left[X_{t+\Delta t}^{w}\right]-\sqrt{\operatorname{Var}\left[X^{w}\right]}\right) / X_{t}^{w}
\end{aligned}
$$

Such parameters are generated for each $\left(D_{i}, S_{i}\right)$.

B. Aggregation into events tree for product-supply chain systems

The overall events tree for each underlying cost, time and quality is created from the events trees of the constituent component-supplier subsystems. We shall discuss the creation of this tree for the cost process. Let $C_{1 t}, C_{2 t}$, and $C_{3 t}$ respectively represent the cost processes for componentsupplier subsystems $\left(D_{1}, S_{1}\right),\left(D_{2}, S_{2}\right)$, and $\left(D_{3}, S_{3}\right)$ at time $t$. The overall cost of the product design and supply chain system $(D, S, E)$ is represented by $C_{t}$ is some aggregation (Aggr) of $C_{1 t}, C_{2 t}$, and $C_{3 t}$ as shown in Figure 3 . At the next state, $t+\Delta t, C_{l t}$ can be at one of the states shown in the trinomial expansion of Figure 2. Thus, $C_{l t}$ can go up by a factor $u_{1}$ or down by a factor $d_{1}$. Similarly, $C_{2 t}$ and $C_{3 t}$ are affected.

In addition, we assume that the jump affects all subsystems simultaneously, i.e., it is not possible that one subsystem jumps while another does not. As a result the aggregate value, $C_{t}$ can take one of nine values at the next time step, $t+\Delta t$. Eight of these states are due to the regular movements (up factor $u_{i}$, or down factor $d_{i}$ ) of each subsystem $\left(D_{i}, S_{i}\right), i \in\{1,2,3\}$. The ninth state, shown as the bottommost state in the figure, is one in which the cost of every subsystem jumps. In the component-supplier event tree, the probability of regular up or down was $(1-\lambda \Delta t) * 0.5$. Therefore, the probability of each of the eight states is (1$\lambda \Delta t)^{*} 0.125$. The probability of the jump state is $\lambda \Delta t$.

\section{Capturing the variations of the manufacturer's expectation}

As explained in Section IV, the real options analysis is carried out along the events tree that captures the variation of the manufacturer's expectation. However, the events on this expectation tree must match the events on the overall 
product design and supply chain tree explained in the previous section. These events correspond to the positions (up, down, jump) of the component-supplier subsystems. Thus, the manufacturer's expectation must also have an equivalent of the 9-event tree shown in Figure 3.

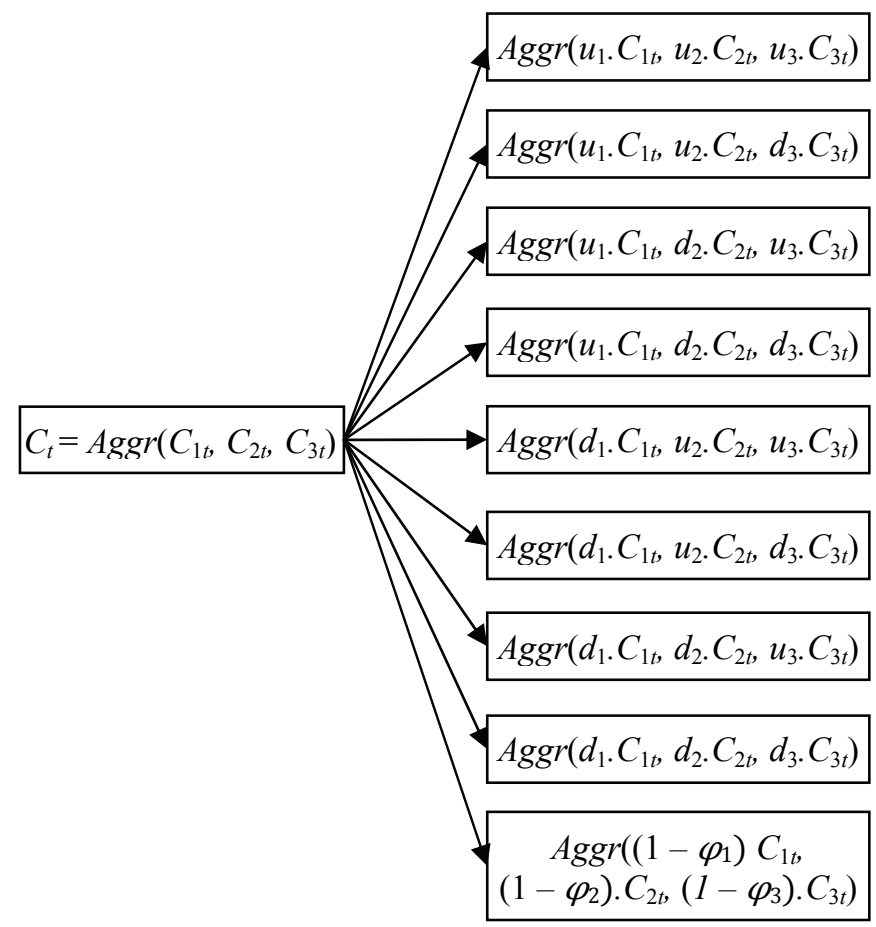

Figure 3 Nine possible events for product and supply chain system in Figure 1.

The manufacturer could use its own knowledge and the knowledge of the processes to generate such a tree. However, this problem is non-trivial, and we are not aware of approaches to address it. In this research, we assume that the manufacturer has internal data on its expectations from each component-supplier subsystem and then aggregates those using the procedure explained in the previous section. This aggregation then forms the tree that corresponds to the manufacturer's expectation.

\section{AGgREGATION: UNIQUE CHARACTERISTIC OF QUALITY}

Whereas the overall cost and time can be deduced from the topology of the supply chain (and they are usually simple summations), the overall product quality depends heavily on the product design and the corresponding manufacturing process. In [10], the geometric weighted mean is presented as an aggregation function for quality. Aggregation functions for evaluating overall performance of the supply chains associated to a global objective whose break-down provides elementary objectives are developed in [11]. The weighted mean aggregation functions are proposed to handle hierarchical links within supply chains.

However, we observe that product quality in the product design and supply chain system has unique characteristics compared to cost and time:

- Component design can alter the relationship between the product quality and the component qualities

- Design changes in downstream supplier enforce design changes in the upstream supplier, which in turn affects overall product quality.

In order to illustrate these points, let us consider a twopart sheet metal assembly in, where suppliers $S_{1}$ and $S_{2}$ manufacture components 1 and 2 (in designs $D_{1}$ and $D_{2}$ ) as shown in Figure 4. Supplier $S_{3}$ receives these components and joins to complete assembly $D_{3}$. This supply chain has been represented as a directed graph in Figure 1.

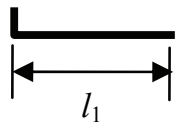

(a)

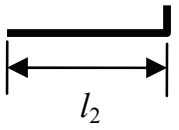



(b)
Figure 4 Two part sheet metal assembly (lap joint): (a) before and (b) after assembly. Product length does not depend on component length.

Let us assume the qualities of each component and the complete assembly are measured as the closeness to their target lengths $l_{1}, l_{2}$, and $l_{3}$, respectively. A standard practice in dimensional tolerancing [12] is to represent the quality as a reciprocal of the tolerance, i.e., $Q_{k}=1 / \Delta l_{\mathrm{k},} k \in\{1,2,3\}$ where $\Delta l_{k}$ is the tolerance (allowable deviation from the target length) of component $k$. Since components 1 and 2 are joined with a lap joint that allows the adjustment of the length of the resulting assembly, quality $Q$ of the entire supply chain depends only on quality $Q_{3}$ of assembly operation (more specifically, the tolerance of the fixture) at $S_{3}$, i.e.,

$$
Q=\operatorname{Aggr}\left(Q_{1}, Q_{2}, Q_{3}\right)=Q_{3}
$$

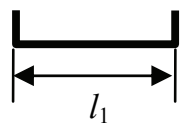

(a)

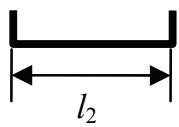

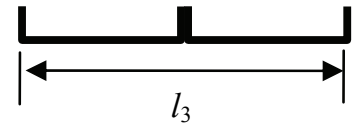

(b)
Figure 5 Two part sheet metal assembly (butt joint): (a) before and (b) after assembly. Product length is the sum of component lengths.

Let us now assume that suppliers 1 and 2 have different designs, $D^{\prime}{ }_{1}$ and $D^{\prime}{ }_{2}$ as shown in Figure 5. Since components 1 and 2 have a butt joint that does not allow adjusting the length of the resulting assembly, the quality $Q$ of the entire supply chain depends only on $Q_{1}$ and $Q_{2}$ (assuming a "thin" weld). The tolerance of the resulting assembly is $\Delta l_{1}+\Delta l_{2}$ due to tolerance stackup [12]. Therefore, $1 / Q=1 / Q_{1}+1 / Q_{2}$, namely,

$$
Q=\operatorname{Aggr}\left(Q_{1}, Q_{2}, Q_{3}\right)=1 /\left(1 / Q_{1}+1 / Q_{2}\right)
$$

On the other hand, the cost and time of the supply chain are given for both cases by

$$
\begin{gathered}
C=\operatorname{Aggr}\left(C_{1}, C_{2}, C_{3}\right)=C_{1}+C_{2}+C_{3} \\
T=\operatorname{Aggr}\left(T_{1}, T_{2}, T_{3}\right)=T_{3}+\max \left\{T_{1}, T_{2}\right\}
\end{gathered}
$$

The following section presents results from the application of our approach to the above two cases. 


\section{CASE STUdY}

Using the two cases (Figure 4 and Figure 5) presented in the previous section, this section explores how the different aggregation functions respond under severe disruptions.

For the sake of simplicity, we assume that $\left(D_{1}, S_{1}\right)$ has an alternative $\left(D_{1}{ }^{\prime}, S_{1}\right)$, and $\left(D_{2}, S_{2}\right)$ has an alternative $\left(D_{2}{ }^{\prime}, S_{2}{ }^{\prime}\right)$, whereas the $\left(D_{3}, S_{3}\right)$ has no alternative. The ability to meet the quality, time, and cost requirements are captured through geometric mean-reverting jump diffusion processes given by Equation (6), with the parameters as shown in Table 1. For simplicity, we also assume that the jump size for each underlying process for each component-supplier subsystem is the same, e.g., for $\left(D_{1}, S_{1}\right)$, each of $C, T$, and $Q$ have the same jump size, 0.25 .

Table 1 Parameters for the processes for each component-supplier subsystem.

\begin{tabular}{|c|c|c|c|c|c|c|}
\hline & $\begin{array}{l}\text { Cost } \\
\text { Ability }\end{array}$ & $\begin{array}{l}\text { Time } \\
\text { Ability }\end{array}$ & $\begin{array}{l}\text { Quality } \\
\text { Ability }\end{array}$ & $\begin{array}{l}\text { Jump } \\
\text { size } \varnothing)\end{array}$ & $\begin{array}{c}\text { Mean } \\
\text { reverting } \\
\text { ratio } \alpha\end{array}$ & $\begin{array}{c}\text { Variance } \\
\text { ratio } \beta\end{array}$ \\
\hline$\left(D_{3}, S_{3}\right)$ & 80 & 80 & 70 & 0.15 & $1.25 \%$ & $15 \%$ \\
\hline$\left(D_{1}, S_{1}\right)$ & 105 & 105 & 95 & 0.65 & $1.25 \%$ & $75 \%$ \\
\hline$\left(D_{1}{ }^{\prime}, S_{1}{ }^{\prime}\right)$ & 105 & 95 & 50 & 0.25 & $3.75 \%$ & $25 \%$ \\
\hline$\left(D_{2}, S_{2}\right)$ & 95 & 105 & 95 & 0.65 & $1.25 \%$ & $75 \%$ \\
\hline$\left(D_{2}{ }^{\prime}, S_{2}{ }^{\prime}\right)$ & 95 & 95 & 50 & 0.25 & $3.75 \%$ & $25 \%$ \\
\hline
\end{tabular}

In order to generate the manufacturer's expectation tree (Section V.C), we assume that the manufacturer has internal data on its expectations from each component-supplier subsystem and then aggregates those using the following equation:

$$
\begin{aligned}
& Q=1 /\left(1 / Q_{1}+1 / Q_{2}+1 / Q_{3}\right) \\
& C=C_{1}+C_{2}+C_{3} \\
& T=\max \left\{T_{1}, T_{2}\right\}+T_{3}
\end{aligned}
$$

Parameters for these expectations are shown in Table 2 and correspond to the geometric mean-reverting process from Equation (6).
Table 2 Manufacturer's expectations from each component-supplier subsystem.

\begin{tabular}{|c|c|c|c|c|c|c|}
\hline $\begin{array}{c}\text { Mean } \\
\text { of Cost } \\
\text { Ability }\end{array}$ & $\begin{array}{c}\text { Mean of } \\
\text { Time } \\
\text { Ability }\end{array}$ & $\begin{array}{c}\text { Mean of } \\
\text { Quality } \\
\text { Ability }\end{array}$ & $\begin{array}{c}\text { Jump } \\
\operatorname{size}(\varnothing)\end{array}$ & $\begin{array}{c}\text { Mean } \\
\text { reverting } \\
\text { ratio } \alpha\end{array}$ & $\begin{array}{c}\text { Variance } \\
\text { ratio } \beta\end{array}$ \\
\hline$(D, S)$ & 80 & 80 & 70 & 0.15 & $1.25 \%$ & $15 \%$ \\
\hline
\end{tabular}

This gives us the following 6 executable alternatives to choose from along the decision tree:

- Alternative 0: Hold the decision

- Alternative -1: Abandon the project at a salvage value of $A=90$

- Alternative 1: Keep current system, i.e., $\left\{\left(D_{1}, S_{1}\right),\left(D_{2}\right.\right.$, $\left.\left.S_{2}\right),\left(D_{3}, S_{3}\right)\right\}$ at no additional cost

- Alternative 2: Change to $\left\{\left(D_{1}{ }^{\prime}, S_{1}{ }^{\prime}\right),\left(D_{2}, S_{2}\right),\left(D_{3}, S_{3}\right)\right\}$ at a switching cost of $K_{2}=5$

- Alternative 3: Change to $\left\{\left(D_{1}, S_{1}\right),\left(D_{2}{ }^{\prime}, S_{2}{ }^{\prime}\right),\left(D_{3}, S_{3}\right)\right\}$ at a switching cost of $K_{3}=5$

- Alternative 4: Change to $\left\{\left(D_{1}{ }^{\prime}, S_{1}{ }^{\prime}\right),\left(D_{2}{ }^{\prime}, S_{2}{ }^{\prime}\right),\left(D_{3}, S_{3}\right)\right\}$ at a switching cost of $K_{4}=10$ (twice the previous ones), since two subsystems have changed.

Figures 6 and 7 show the partial optimal decision tree from the computation for the lap joint and butt joint, respectively shown in Figures 4 and 5. Labels on the edges indicate a decision that the manufacturer should undertake at that node. Interesting results are found in the shown extremities, and they help us judge how extreme cases how different aggregation functions for quality affect to the optimal decision.

In Figure 6, it is observed that the decision is a "Hold" in jump node (leftmost node) at the time $t=1$, which represents that delaying the decision is the best choice until the next events are revealed. On the other hand, the rightmost node at the time period $t=1$ indicates that a decision is to continue with Alternative 1 . At the next time step $(t=2)$ branching from the rightmost node at $t=1$ the optimal decision is to "switch" from the alternative 1 to 3 rather than abandoning the project when disruptive event occurs. The interesting node is the leftmost node at the time period $t=2$, because decision represents to switch to the Alternative 4 after two consecutive jumps. The alternative 4 is chosen

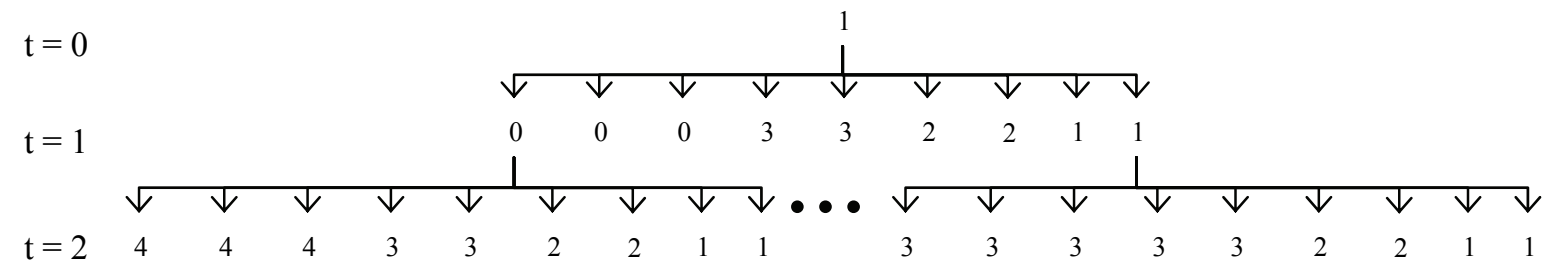

Figure 6 An optimal decision tree of case 1 (Figure 4). At each node, the value of the decision variable is shown.

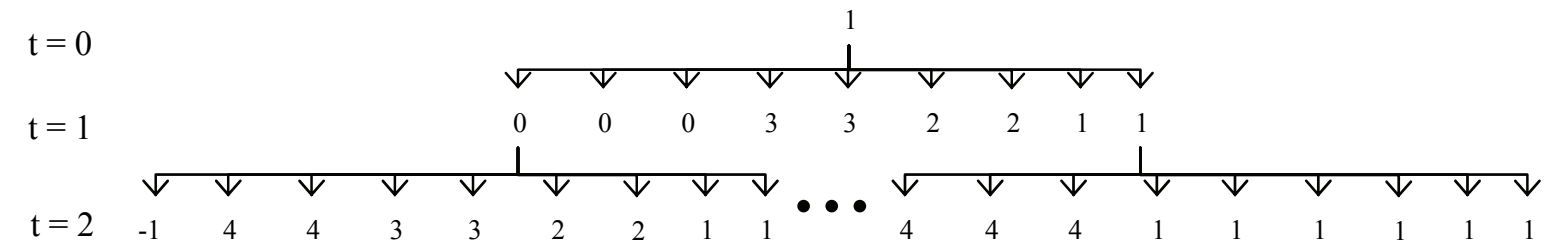

Figure 7 An optimal decision tree of case 2 (Figure 5). At each node, the value of the decision variable is shown. 
because it is resistant to disruptions. The performance values of the alternative 4 drop slightly with a small $\varnothing$, as show in Table 1. Note that suppliers $S_{1}{ }^{\prime}$ and $S_{2}$ ' of the alternative 4 have fairly low values for quality as shown in Table 1; however, in the case of the lap joint, the aggregated quality value is not affected by the values of these two suppliers.

Figure 7 shows the results for the case when components 1 and 2 are joined with a butt joint. Therefore quality $\mathrm{Q}$ of the entire supply chain now depends on $Q_{1}$ and $Q_{2}$. The performance values of aggregated qualities then have lower value than a lap joint case in most of the cases, because aggregation function is different. As a result, overall performance values are also decreased compared to the previous example. The decision is "Abandon" at the leftmost node at the time period $t=2$, where jump disruptions occur (compared to the decision of moving to Alternative 4 in Figure 6). However, Alterative 4 becomes more preferable choice at the nodes where disruptive events occur as shown in Figure 7 (compared to case in Figure 6, where it was Alternative 3).

\section{CONCLUSION}

Understanding how high-impact uncertain events would affect the enterprise is of paramount importance. Research efforts have traditionally focused on using single criteria, such as costs (and more recently, lead-time) as the criteria for evaluating the supply chain. In this paper, we focus on the simultaneous synthesis of product design and supplychain to handle adaptation when variation in the operating environment is beyond the robustness limits. We focus on product quality (design criteria) along with cost and lead time (supplier attributes) as drivers of the performance, by modeling them as mean-reverting jump-diffusion processes.

We have demonstrated the application to a twocomponent two-echelon product design and supply chain system. In particular, we have shown how product quality has very unique characteristics and requires special handling in the process of deciding to adapt.

Our ongoing work attempts to address the following issues:

1. The events and decision trees are quite large even with a small number of alternatives and time steps, making their interpretation a challenge.

2. Creating the event tree to capture variations of the manufacturer's expectations under the constraint that it should match real events of the component-supplier subsystems is a non-trivial task.

3. While the work presented in this research assumes that the need to adapt, predicting such a need and guessing the parameters of the corresponding processes requires appropriate data collection and analysis from all the stakeholders.

4. Most jumps that affect product design are nonMarkovian. Currently, the use of Poisson process means that a jump could (although, with a low probability) repeat in consecutive time steps.

5. The scenarios of lap joint versus that of butt joint as should not be considered as independent. In this paper, the independence is consistent with the initial assumption that every alternative component-supplier subsystem is an exact replacement for the existing one, and it does not impact other subsystems. However, there is a need to consider the two cases as alternatives within one larger and explore how changing one componentsupplier subsystem could force another one to be changed.

\section{REFERENCES}

[1] L. Patil and K. Saitou, "Adapting product and supply-chain systems under severe uncertainty," in IEEE Conference on Automation Science and Engineering (CASE '08) Washington D.C., 2008.

[2] Y. Sheffi, The resilient enterprise: Overcoming vulnerability for competitive advantage. Cambridge, MA: MIT Press, 2005.

[3] A. K. Dixit and R. S. Pindyck, Investment under uncertainty. Princeton, N.J.: Princeton University Press, 1994.

[4] R. de Neufville and T. Wang, "Building Real Options into Physical Systems with Stochastic Mixed-Integer Programming," in Proceedings of the 8th Annual International Conference on Real Options Montreal, Canada, 2004.

[5] H. Yao, J. Li, and L. E. Holloway, "Towards modeling of resilience dynamics in manufacturing enterprises: Literature review and problem formulation," in IEEE Conference on Automation Science and Engineering (CASE '08) Washington D.C., 2008.

[6] L. V. Snyder, "Supply Chain Robustness and Reliability: Models and Algorithms," in Department of Industrial Engineering and Management Sciences. vol. Ph.D. dissertation Evanston: Northwestern University, 2003.

[7] D. Garg, Y. Narahari, and N. Viswanadham, "Design of six sigma supply chains," IEEE Transactions on Automation Science and Engineering, vol. 1, pp. 38-57, Jul 2004.

[8] R. S. Gaonkar and N. Viswanadham, "Analytical Framework for the Management of Risk in Supply Chains," IEEE Transactions on Automation Science and Engineering, vol. 4, pp. 265-273, 2007.

[9] R. Cont and P. Tankov, Financial Modelling with Jump Processes: Chapman and Hall/CRC, 2007.

[10] E. S. Scott, "Axiomatic Justification for a Geometric Quality Aggregation Function," Decision Sciences, vol. 30, pp. 415-440, 1999.

[11] L. Berrah and V. Clivill, "Towards an aggregation performance measurement system model in a supply chain context," Computers in Industry, vol. 58, pp. 709-719, 2007.

[12] C. M. Creveling, Tolerance design : a handbook for developing optimal specifications. Reading, MA: Addison-Wesley, 1997. 